was great reason to believe that the medulla spinalis was severely injured. He did not complain of pain or uneasiness, but of numbness of his legs, and slight difficulty in breathing. His head was slightly lacerated in several places. The next morning the following was ordered:-Chloride of mercury, two grains; powdered opium, lialf a grain. Mix, for a pill at night. Castor oil, one ounce, in the morning. The urine to be drawn off by the catheter.

Oct. 13th.-Has perspired copiously during the night, and says that he is free from pain. Bowels have been opened by the medicine; considerable thirst; pulse rather full; tongue clean; urine scanty and alkaline; no medicines.

14th.-Has passed a very restless night, and has great weight and pain in the chest, with a short dry cough. Tongue white; skin hot; pulse very frequent, strong, and full; urine high coloured. Ordered calomel, two grains, with half a grain of opium, every night.- Evening, eight o'clock: About midday the symptoms became much aggravated; pulse rapid and strong; twelve ounces of blood were then ordered to be taken from the arm, which slightly lowered the pulse. Soon after the bleeding, the patient brought up, by retching, a considerable quantity of mucus from the throat, and then became very languid. A little wine was ordered to be given to him from time to time.-Ten o'clock: The patient, since last report, has become slightly delirious, and is talking incoherently; the pain in the chest continues. Ordered twelve leeches to the chest; tincture of opium thirty minims, to be taken immediately. The catheter has been introduced, and the urine drawn off four times every day since his admittance into the hospital.

15th.-The patient passed a very restless night, and, at half-past seven o'clock this morning, he died.

Autopsy, seventeen hours after death.-Lungs greatly congested, in several places hepatized; bladder quite healthy and filled with urine; the seat of the injury which proved fatal was found in the spine, at the sixth dorsal vertebræ, the spinous process of which was broken in two, with a comminuted fracture, and very slight lateral displacement of the body. There was also laceration of the posterior common ligament; the spinal cord exhibited the following appear ances: extravasation of blood below the injured part externa to the sheath; inflammation of the cord, extending from the fractured vertebra upwards; the sheath marked about the seat of the injury with delicate green patches.

Observations on the three cases.-The above cases exhibit some of the varying phases of symptoms following fractures of the spine, although their essential characteristics are very similar. It should be remarked, that in two of the cases there were not any well-marked evidences of displacement of the bone at the time of admission, and consequently in them surgery could not do more than administer to the paralysis which existed, and the local inflammation at the seat of injury. With respect to the third case, in which displacement existed, it is questionable whether means of replacement should be adopted. From examining the ordinary effects of fracture upon the medulla, it must be evident that, crushed and broken down as it usually is, the mere removal of pressure from it will not restore its function in the greater number of cases, and therefore it may be said that little good is likely to arise from any attempt in that direction. Yet, on the other hand, there are some few cases in which the medulla does not suffer to the extent indicated; but merely from pressure, without breaking down of its structure. In such cases, replacement of a displaced bone, and consequent removal of pressure from the medulla spinalis, presents a great probability of affording effectual relief to the paralysis and recovery to the patient. Now, as by the symptoms there is not any positive indications by which the less can be distinguished from the greater degrees of lesion, it appears reasonable that, when displacement exists, as ascertained by irregularities in the course of the spinous processes, means should be used in all cases, especially as there are sufficient safeguards in the use of those means against any further injury occurring therefrom. By thus proceeding we may afford relief in some few cases, and we certainly do not place the remainder in a worse position.

\section{ON THE TYPES OF DISEASES OF THE SPINAL SYSTEM PRESEN'TED BY EXPERIMENTS.}

Bx MARSHALL HALL, M.D., F.R.S., \&c.

ONE of the great advantages of experimental researches in the spinal system is, that they frequently present TYPEs of its diseases; and the great advantage of these is, that they pre- sent the opportunity for the investigation of the nature of those diseases, and the deduction of their remedies.

The diseases of the spinal system exist under several forms which admit generally of being reproduced in experiments for the purpose, and with the objects to which I have just adverted.

In general the diseases of the spinal system occur under the form of spasm, of paralysis, or of the two combined-viz. spasmo-paralysis. They are all primarily affections of the excito-motor muscular system, to the exclusion of the sentient or cerebral system-a singular confirmation of a physiological doctrine, that these two systems are totally distinct from each other.

Generally spasm consists in iritation of nervous tissue, stil retaining its normal structure, whilst paralysis implies a lesion of that structure. But the structure of the nervous tissue must be viewed under several aspects: thus this tissue may be injured by being lacerated or bruised at any given point; but it may be strangely injured in a given point by injury inflicted at a distance, through the means of shock. In this manner spasm is apt to lead to paralysis, and for the obvious reason that this peculiar lesion is of the most intimate or atomic character, unlike the division or separation of its atoms by laceration, \&c.; the paralysis, or spasmo-paralysis, so induced, is less persistent than in the latter case.

This remark leads me to the subject of the excito-motor power itself, on which all the movements connected with the spinal system depend. This power may be diminished or even suspended in its energies; it may also be greatly augmented, in which case there is proportionate augmented susceptibility to impressions, and to excited actions, the results of those impressions.

The former state of things is induced by all agents of such violent character as to induce shock; the latter is induced by peculiar agents, of a chemical or physical character, which act more gently on the nervous structures. The same identical agent may produce either of these effects, indeed, according to its degree. Thus too large a dose of strychnine speedily destroys all excitability; a very minute dose, on the contrary, induces the most extraordinary phenomena of augmented ex citability which we can witness. The action of the electric current is precisely similar: a very mild current produces purely physiological effects; too strong a current soon induces destruction of the excitability of this part of the nervous system. The first effect of decapitation (of shock) in a frog is a suspension of the excito-motor power; the second and ulterior effect is, or is supposed to be, an augmented susceptibility and activity of this vital agent. Electricity, heat, and the class of agents, called stimuli, generally-may be employed so as to act as augmentive or destructive of the excito-motor nervous power.

As an example of these effects, I may mention, for the benefit of those who have not performed an experiment, that immediately after decapitation of the frog, no reflex action are produced on irritating the foot; it is diminished excitability, the effect of shock. On administering a minute dose of strychnine, on the contrary, the touch, even of a feather, in duces reflex action of a tetanic force and character.

From experiments we learn that this augmented susceptibility or excitability may exist in force withont existing in action. A frog may be under the influence of strychnine yet, if not actually excited, it may remain quiescent, relaxed in posture: it is tetanole, without being tetanic, affording a type and idea of certain diseases of the spinal system, of the deepest interest-one on which the rationale of the symptoms, and the adaptation of remedies, alike and equally depend.

Now, in this point of view, the effect of strychnine on the frog presents the TrPe of hydrophobia. In both the blood is poisoned,-in both the spinal system is in a condition of extremely augmented excitability, without being necessarily excited. Avoid all excitation, and the frog recovers. Apply excitation of the mildest character continuously, and the frog speedily dies. Here an experiment accurately presents, not only the type of the malady, but of the treatment!

In this experiment, as in hydrophobia itself, we have two conditions-one of excitability, the other of actual excitement, according as excitation is arerted or admitted.

I propose to designate the former condition by the termina tion ode, the latter by that of $i c$. The froy, unexcited, is still tetanode; excited, it becomes tetanic. The patient affected with hydrophobia is hydrophobocle; is he necessarily hydro phobic? The former state admits of recovery; the latter soon destroys. I beg my reader to turn to pp. 151 .

In this point of view we may consider other diseases of the spinal system. The patient affected with tetanus is not 
neessarily tetanic; he is only tetanode. If excited, he dies. What if he were, or could be, preserved absolutely from all excitement? He dies of violent and exhausting spasms. These spasms are reflex actions; reflex actions are necessarily excited. Remove all excitation, and the spasms-that is, the cause of death-would be averted.

In like manner the newly decapitated snake is full of excitor and motor power, yet free from movement unless an excitant be applied.

So also of certain forms of convulsive disease in infants and in adults. The patients are spasmatode, or spasmatic, according as they are excited or preserved from excitement.

The difference throughout is that of the disposition to, or actual condition of, spasmodic action, or that of excitability and excitement. The former may subside; the latter destroys !

The further difference between hydrophobia and tetanus is, as my friend, Mr. Simpson, has so well portrayed, in the fourth plate of my "New Memoir," that the former is induced through the medium of the blood; the latter through that of an incident nerve. Of the former the frog affected with strychnine presents the exact TYPE; of the latter I doubt whether we possess an EXACT type in any experiment.

No experiment has been devised, to my knowledge, to induce augmented excitability of the spinal system through the medium of an incident nerve or nerves, or of any part of the spinal system. It is said that the decapitated batrachian is more excitable than the entire animal. But $I$ think this a mistake. The effects of excitation are controlled by efforts of volition in the perfect animal, and uncontrolled in the decapitated animal. The difference is rather apparent than real.

Thus of traumatic tetanus, I believe I may affirm that we have not yet an experimental type. This is to be regretted the more because many questions arise which can only be speedily determined by means of experiment. For example, do the spasms perfectly relax during the course of the disease? The trismus seems persistent-is it so? Or does it cease and return on any excitement from volition, emotion, or excitation of reflex action? Does an act of volition, exclusive of emotion and of reflex action, pass into spasm? As in the stammerer, the attempt to speak passes into excited action; so in trismus, the attempt to open the mouth closes the jaws more firmly. Volition passes into excited action.

I fear that in extreme cases the acts of respiration and of deglutition may pass into general spasm; so that to avoid all morbid action and its consequences may be impossible. Still, the type of the disease in its augmented excitability, and the principle to guide us in the treatment-that of avoiding all excitation-are set before us in an experiment; and though the spasm may never cease absolutely, it will not be the less essential to avoid all causes of its exasperation.

It is to be observed, too, that in all experiments on the batrachian tribes, it is only types and principles which are presented to us, and on which our office is to reason correctly, and perhaps to experiment in our turn. It was an experi ment on the frog which suggested to me the only hopeful way of treating hydrophobia and tetanus!

In dentition there is a state of angmented excitability and actual excitement, arising, as in tetanus, from injury done to nerve. I have not, as I have already said, seen augmented excitability in any experiment consisting of injury inflicted on nervous tissue.

I now come to a new series of types of disease of the spinal system. They consist in actual excitement of its different parts, the incident nerves, the spinal centre, and the muscula nerves, portrayed in a series of experiments on electrogenic states of these living tissues, laid before the Royal Society, but not understood by those who pretended to judge them there.

The electrogenic state is that induced in a nervous structure by the continuous passage of a current of galvanism, of a force in due physiological relation to the excitability of the animal. Its phenomena are observed on withdrawing this agency.

The effect of this electrogenic state on the incident nerves is seen in varied reflex actions. The experiment is a type of those convulsive maladies which consist in excited reflex actions, such as are seen in dental, gastric, intestinal, and uterine irritation.

The electrogenic condition of the spinal marrow itself is the type of the class of certain convulsive affections which arise from arachnitis at the base of the brain, spinal arachnitis, \&c.

Lastly, the electrogenic condition of the muscular nerves is the type of those cases of spasmodic affection arising from neuritis, or of inflammation of the neurilemma. Such a state of things exists in the second or convalescent stage of facial paralysis. The tumefaction of the neurilemma having subsided, the neurine is irritated instead of being compressed, and spasm is the consequence. In the experiment of the electrogenic condition of a muscular nerve, the frequent discharge of the electrogenic state produces a similar effect on the nervimuscular fibre.

There is still another class of TrPES of diseases of the nervous system displayed in our experiments. The cerebrum and cerebellum being removed in a dog, extraordinary reflex actions are induced by irritating the dura mater at various points.

Having removed the head and sternum in a turtle, various reflex movements are produced by tearing away the viscera.

It is obvious that irritation of the internal membranes and tissues may thus be a source of reflex convulsive maladies; that of the cerehral membranes induces various convulsive or tetanoid affections; so does irritation of the serous membranes within the thorax and abdomen,-especially that of the pericardium.

It is but a step from these affections to those dependent on dorsal, gastric, intestinal, and nterine irritation, with which we are so familiar.

It is but a step further to consider the remarkable effects occasionally attendant on passing a bougie or catheter, on irritating the meatus externus of the ear, on the passing of a biliary or renal calculus, \&c. One patient experiences a sort of rigor, and an irrepressible deep inspiration on voiding the bladder, when that act has been too long delayed. Experiments might imitate some of these morbid phenomena.

I will conclude these observations by adverting to a question full of the deepest interest. A certain class of intracranial diseases-seated, I believe, at the base, and adjacent to the medulla oblongata-is attended with protracted sickness and vomiting; tetanoid, or epileptoid spasm; perhaps priapism and sexual excitement. What is the precise rart, or organ, irritated in such cases? Experiment alone can teach us. I propose to place animals-dogs, for exampleunder the influence of chloroform, and to perform a series of experiments, to determine these and other important questions, replete as they would be with diagnostic, and, consequently, with practical suggestions.

It is in this manner only that irritation of the cerebrum, of the corebellum, of the medulla oblongata, of certain nerves within the cranium, as the nerves of special sense, the trifacial, the facial, \&c., the several membranes and their subdivisions, can be accurately and distinctly ascertained. When so ascertained, their application, as types, to the diagnosis of diseases, will be as obvious as it is important.

One such experiment I performed long ago.* Myaversion to the intiction of pain prevented me from ever repeating it valuable as its results appeared. The discovery of the tnæsthetic effects of ether, or of chloroform, will shed a benefit upon experimental physiology.

There is another experiment of great interest. In endeavouring to procure the circulation in the web of the frog independently of the spinal marrow, $I$ always found that the most careful destruction of the medulla oblongata was attended by its annihilation. This organ exerts, therefore, extreme influence on the action of the heart. Is it on this account that a state of pallor, so like that of syncope, occurs in many attacks of the apoplectic and epileptic character, in seasickness, in other forms of sickness, \&c.

All this is a part of scientific medicine and surgery. I believe it was an early career of experiment which made our greatest surgeon what be is. He sees symptoms by the light of physiology, and understands them! How apt and how beautiful, and how peculiarly his own, were his illustrations of surgical disease, on all occasions, from the chair of the Royal Medical and Chirurgical Society!

Manchester-square, August, 1848 .

ON A

NEW MODE OF TREATING DEAFNESS,

ATTENDED BY LOSS OF THE MEMBRANA TYMPANI, ASSOCTATED, OB NOT, WITH DISCHARGE FROM THE EAR.

By JAMES YEARSLEY, EsQ., M.R.C.S., SURGEON to THE METROFOLITAN EAR iNSTITUTION. (Continued from p. 155.)

THE operation of perforation of the membrana tympani, when first introduced as a mode of removing deafness, was performed by Sir Astley Cooper in two kinds of cases-those * See my Observations and Suggestions, Series ii. p. 64 . 